Z Rheumatol $2021 \cdot 80: 588-589$

https://doi.org/10.1007/s00393-021-01033-z

Published online: 6 July 2021

(c) Springer Medizin Verlag $\mathrm{GmbH}$, ein Teil von

Springer Nature 2021

\section{Erratum to: Diagnostic value of the CD 15 focus score in two- stage revision arthroplasty of periprosthetic joint infections. High specificity in diagnosing infect eradication}

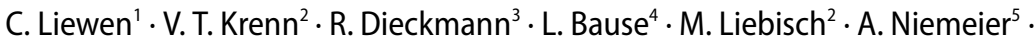 \\ A. Trampuz ${ }^{6} \cdot$ V. Krenn ${ }^{1}$ \\ ${ }^{1}$ MVZ-Zentrum für Histologie, Zytologie und Molekulare Diagnostik Trier GmbH, Trier, Germany \\ ${ }^{2}$ Fakultät für Medizin, Sigmund Freud PrivatUniversität Wien, Vienna, Austria \\ ${ }^{3}$ Krankenhaus der Barmherzigen Brüder Trier, Trier, Germany \\ ${ }^{4}$ St. Josef-Stift Sendenhorst, Sendenhorst, Germany \\ ${ }^{5}$ Department of Orthopedics, University Medical Center Hamburg-Eppendorf, Hamburg, Germany \\ ${ }^{6}$ Center for Musculoskeletal Surgery (CMSC), Charité-Universitätsmedizin Berlin, Berlin, Germany
}

\section{Erratum to:}

Z Rheumatol 2020

https://doi.org/10.1007/s00393-020-

00941-w

Dear Reader,

In the original online version of the article, - Fig. 2 was incorrect. Please refer to the corrected • Fig. 2.

The original article has been corrected.

\section{Corresponding address}

Prof. Dr. med. V. Krenn

MVZ-Zentrum für Histologie, Zytologie und Molekulare Diagnostik Trier GmbH Max-Planck-Straße 5, 54296 Trier, Germany Krenn@patho-trier.de 


\section{Modified Consensus Classification of Endoprosthetic Pathology Type I-Type IX}



Fig. $2 \Delta$ Proposal of the revised SLIM/synovitis classification 